The Be Phenomenon in Early-Type Stars, IAU Colloquium 175

ASP Conference Series, Vol. 214, 2000

M. A. Smith, H. F. Henrichs, and J. Fabregat, eds.

\title{
Optical and Near-Infrared Spectroscopy of the Emission Line Young Stellar Object Gy 2-18 (HHL 31)
}

\author{
Paolo Persi ${ }^{1}$, Vito Francesco Polcaro ${ }^{1}$, Joaquin Bohigas ${ }^{2}$, Mauricio \\ Tapia $^{2}$ \\ ${ }^{1}$ Istituto di Astrofisica Spaziale, CNR, Roma, Italy \\ ${ }^{2}$ Instituto de Astronomia UNAM, Apdo Postal 877, Ensenada, B.C. \\ Mexico
}

\begin{abstract}
We present the results of optical and near-IR spectroscopy of the massive young star associated with the extended reflection nebula Gy 2-18 (HHL31). The optical spectrum between 4000-5000 $\AA$ suggests a B0-B2 classification for this object. In addition the $\mathrm{H} \alpha$ line profile indicate the presence of a consistent stellar wind with a velocity of the order of $1200 \mathrm{~km} / \mathrm{s}$. The near-IR spectrum is dominated by the presence of the hydrogen recombination emission lines (Pfund and Bracket series). The measured line intensities of these lines are in agreement with the presence of a strong stellar wind in Gy2-18.
\end{abstract}

Gy 2-18 is a peculiar optical object found by Gyulbudaghyan (1983). It is located in a dense cloud detected in CO by Wouterloot \& Brand (1989). $J H K$ images show a young embedded cluster composed at least of 9 very young massive members centered around a point-like central condensation with a bright $\mathrm{H} \alpha$ in emission (Tapia et al. 1997) (see Figure 1). In order to understand the nature of this point-like condensation, we have obtained optical and near- IR spectroscopy.

Two low resolution spectrograms of Gy 2-18 in the 4000-7800 $\AA$ range with $\Delta \lambda=5.5 \AA$ were taken in January 1996 at the Loiano $1.52 \mathrm{~m}$ Telescope with the Bologna Faint Objects Spectrometer and Camera (BFOSC, Merighi et al. 1994) and a Thompson $1100 \times 1100 \mathrm{CCD}$. Four further spectra were taken in January 1998 with a new $2048 \times 2048 \mathrm{CCD}$, widening our spectral range to $3500-9000 \AA$. Spectra of the standard stars Feige 34 and Hiltner 600 were taken in both runs with the same instrumental set-up and were used for absolute flux calibration. Data were then reduced and analyzed using standard IRAF procedures.

The E-W section of the spectrogram at the $\mathrm{H} \alpha$ wavelength shows a diffuse emission elongated 35 arcsec; the point source is located 5 arcsec East from the center of the $\mathrm{H} \alpha$ region (see Fig. 2). From our flux calibrated spectra we can evaluated $V=18.8$ magnitudes for this stellar object. Its spectrum shows a strong $\mathrm{H} \alpha$ emission with equivalent width equal to $-266 \pm 31 \AA$. The line profile is not completely gaussian, thus we can deduce the possible presence of a $\mathrm{P}$ Cygni profile. The line is quite broad $(\Delta \lambda=45 \AA)$.

If interpreted as a Doppler broadening, this widening corresponds to a wind velocity of $1200 \pm 172 \mathrm{~km} / \mathrm{s}$, that is compatible with a late $\mathrm{O}$ or early $\mathrm{B}$ spectral type (see, e.g., Hanuschik, 1996). A precise spectra classification of the object 


\section{GY 2-18 K-Band}

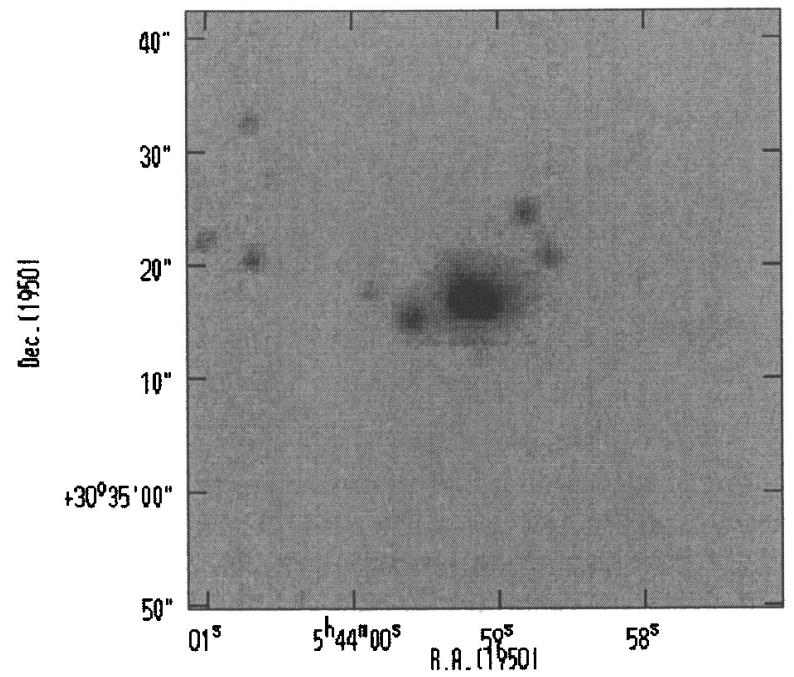

Figure 1. K-band image of Gy 2-18

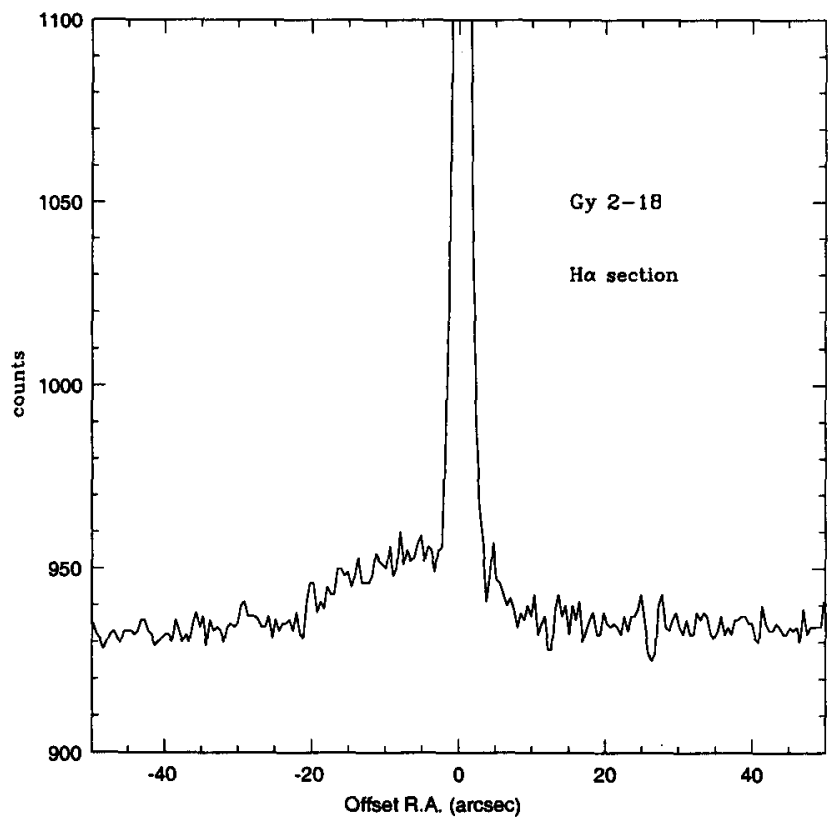

Figure 2. E-W section of the Gy 2-18 spectrogram at the H $\alpha$ wavelength 

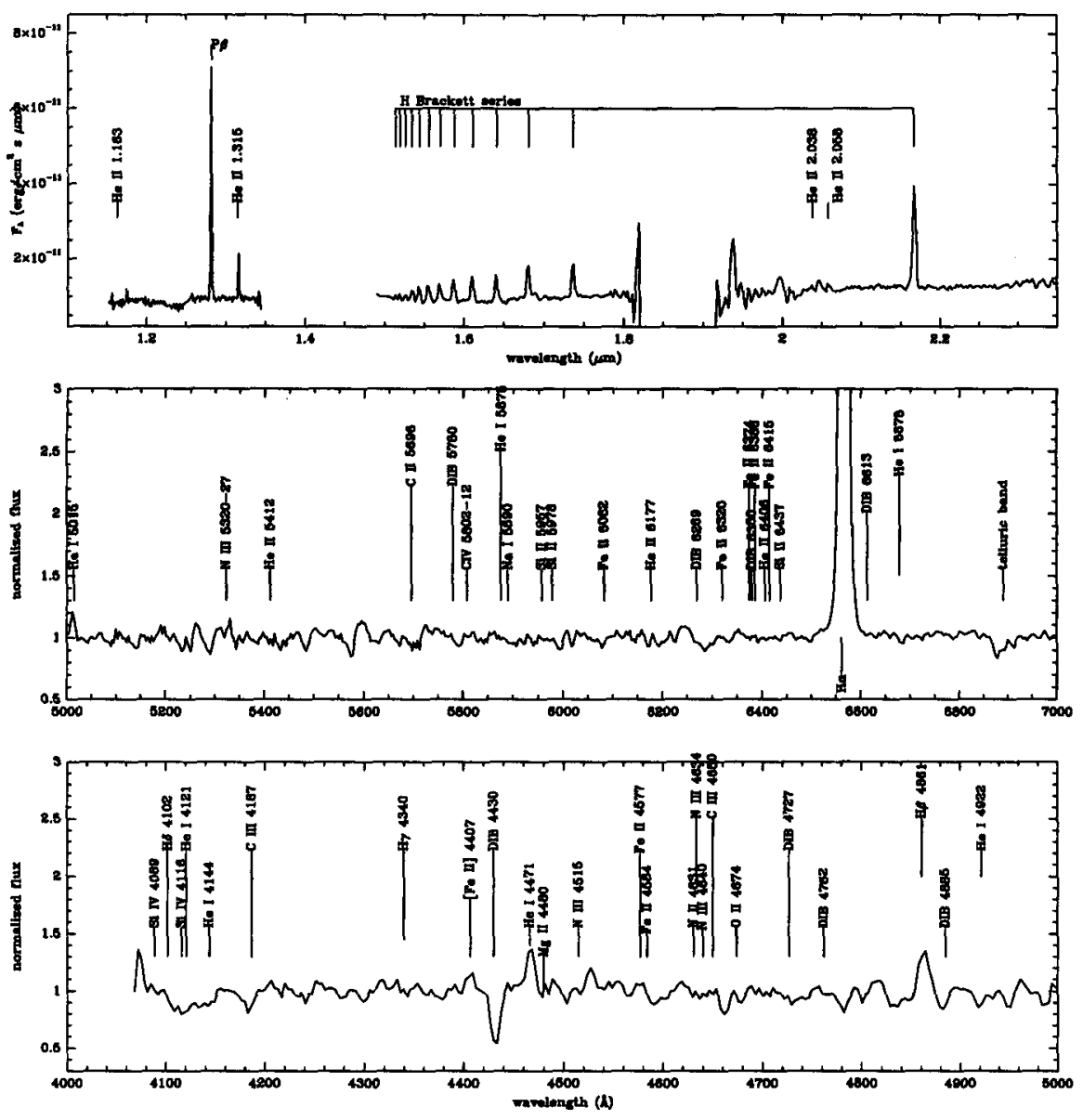

Figure 3. Optical and IR spectrum of Gy 2-18

is of course difficult, both because of the many emission lines, that can mask the true photospheric features and because of the very low flux. In order to solve the last problem and rise the $\mathrm{S} / \mathrm{N}$, we have coadded all the four spectra taken in 1998, Fig. 3 shows the resulting 4000-9000 $\AA$ normalized spectrum of the stellar component. A number of He I, N II, N III, C III, Si IV lines are clearly visible. We can thus confirm an early B (BO-B2) spectral classification (see, e.g., Jaschek \& Jaschek, 1987). A few Fe II and [Fe II] lines are also visible in emission, as well as some He I lines. We can thus suggest that we are dealing with an early main sequence star. If this spectral classification is correct, a $T_{\text {eff }} \simeq 20000 \mathrm{~K}$ must be assumed. In order to fit the measured continuum with such a temperature, an $E(B-V)=2.5$ must be assumed.

The near-IR spectrum between 1 and $2.5 \mu \mathrm{m}$ using a $256 \times 256$ NICMOS3 array, attached at the $2.1 \mathrm{~m}$ telescope was obtained on October 1996 with the spectro-camera CAMAELEON of the Mexican National Observatory of S. Pedro 
Martir (Baja California). The slit was oriented E-W direction with a size of $22^{\prime \prime} \times 0.75^{\prime \prime}$ arcsec. The spectral resolution was $\mathrm{R} \simeq 500$. The spectrum (see Fig.

3 ) is dominated by the hydrogen Brackett series with strong Br- $\gamma$ and Pfund $\beta$ hydrogen emission recombination lines. Their measured line intensities are in agreement with the presence of a strong stellar wind. In addition, the spectrum shows the He II emission lines at 1.163, 1.315 and $2.038 \mu \mathrm{m}$, and the weak He I emission line at $2.058 \mu \mathrm{m}$.

A model to derive the mass-loss rate from the hydrogen recombination lines is in progress.

In summary, we have identified the central object of the young stellar cluster in Gy 2-18 as a massive optically visible B0-B2 star with a strong stellar wind.

\section{References}

Gyulbadaghay, A. L. 1983, Sov. Astr. Lett., 8, 123

Hanuschik, R.W. 1996, A\&A 308, 170

Jaschek, C., Jaschek, M. 1987, "The classification of stars", Cambridge Univ. Press

Merighi, R., Mignoli, M., Ciattaglia, C., et al. 1994, RT 09-1994-05, Bologna Astronomical Observatory

Tapia, M., Persi, P., Bohigas, J., Ferrari-Toniolo, M. 1997 AJ 113, 1769

Wouterloot, J.G.A., Brand, J. 1989, A\&AS 80, 149 